\title{
Progress Toward Ignition at the National Ignition Facility
}

D. E. Hinkel, M. J. Edwards, P. A. Amendt, R. Benedetti, L. Berzak Hopkins, D. Bleuel, T. R. Boehly, D. K. Bradley, J. A. Caggiano, D. A. Callahan, P. M. Celliers, C. J. Cerjan, D. Clark, G. W. Collins, E. L. Dewald, T. R. Dittrich, L. Divol, S. N. Dixit, T. Doeppner, D. Edgell, J. Eggert, D. Farley, J. A. Frenje, V. Glebov, S. M. Glenn, S. W. Haan, A. Hamza, B. A. Hammel, C. A. Haynam, J. H. Hammer, R. F. Heeter, H. W. Herrmann, O. Hurricane, N. Izumi, M. Gatu Johnson, O. S. Jones, D. H. Kalantar, R. L. Kauffman, J. D. Kilkenny, J. L. Kline, J. P. Knauer, J. A. Koch, A. Kritcher, G. A. Kyrala, K. LaFortune, O. L. Landen, B. F. Lasinski, T. Ma, A. J. Mackinnon, A. J. Macphee, E. Mapoles, J. L. Milovich, J. D. Moody, D. Meeker, N. B. Meezan, P. Michel, A. S. Moore, D. H. Munro, A. Nikroo, et al.

July 17,2013

European Physical Society Helsinki, Finland July 1, 2013 through July 5, 2013 
This document was prepared as an account of work sponsored by an agency of the United States government. Neither the United States government nor Lawrence Livermore National Security, LLC, nor any of their employees makes any warranty, expressed or implied, or assumes any legal liability or responsibility for the accuracy, completeness, or usefulness of any information, apparatus, product, or process disclosed, or represents that its use would not infringe privately owned rights. Reference herein to any specific commercial product, process, or service by trade name, trademark, manufacturer, or otherwise does not necessarily constitute or imply its endorsement, recommendation, or favoring by the United States government or Lawrence Livermore National Security, LLC. The views and opinions of authors expressed herein do not necessarily state or reflect those of the United States government or Lawrence Livermore National Security, LLC, and shall not be used for advertising or product endorsement purposes. 


\section{Progress Toward Ignition at the National Ignition Facility}

D E Hinkel ${ }^{1}$, M J Edwards ${ }^{1}$, P A Amendt ${ }^{1}$, R Benedetti ${ }^{1}$, L Berzak Hopkins $^{1}$, D Bleuel ${ }^{1}$, T R Boehly ${ }^{2}$, D K Bradley ${ }^{1}$, J A Caggiano', D A Callahan $^{1}$, P M Celliers ${ }^{1}$, C J Cerjan ${ }^{1}$, D Clark ${ }^{1}$, G W Collins ${ }^{1}$, E L Dewald $^{1}$, T R Dittrich ${ }^{1}$, L Divol ${ }^{1}$, S N Dixit ${ }^{1}$, T Doeppner ${ }^{1}$, D Edgell ${ }^{2}$, J Eggert $^{1}$, D Farley ${ }^{1}$, J A Frenje ${ }^{5}$, V Glebov ${ }^{2}$, S M Glenn ${ }^{1}$, S W Haan ${ }^{1}$, A Hamza $^{1}$, B A Hammel ${ }^{1}$, C A Haynam ${ }^{1}$, J H Hammer ${ }^{1}$, R F Heeter ${ }^{1}$, H W

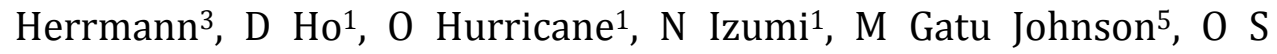
Jones $^{1}$, D H Kalantar ${ }^{1}$, R L Kauffman ${ }^{1}$, J D Kilkenny ${ }^{6}$, J L Kline ${ }^{3}$, J P Knauer $^{2}$, J A Koch ${ }^{1}$, A Kritcher ${ }^{1}$, G A Kyrala ${ }^{3}$, K LaFortune ${ }^{1}$, O L Landen $^{1}$, B F Lasinski ${ }^{1}$, T Ma ${ }^{1}$, A J Mackinnon ${ }^{1}$, A J Macphee ${ }^{1}$, E Mapoles $^{1}$, J L Milovich ${ }^{1}$, J D Moody ${ }^{1}$, D Meeker ${ }^{1}$, N B Meezan ${ }^{1}$, P Michel $^{1}$, A S Moore ${ }^{7}$, D H Munro ${ }^{1}$, A Nikroo ${ }^{6}$, R E Olson ${ }^{4}$, K Opachich ${ }^{1}$, A Pak ${ }^{1}$, T Parham ${ }^{1}$, P Patel ${ }^{1}$, H-S Park ${ }^{1}$, R P Petrasso ${ }^{5}$, J Ralph ${ }^{1}$, S P Regann $^{2}$, B A Remington ${ }^{1}$, H G Rinderknecht ${ }^{5}$, H F Robey ${ }^{1}$, M D Rosen ${ }^{1}$, J S Ross ${ }^{1}$, R Rygg ${ }^{1}$, J D Salmonson ${ }^{1}$, T C Sangster ${ }^{2}$, M B Schneider ${ }^{1}$, V Smalyuk $^{1}$, B K Spears ${ }^{1}$, P T Springer ${ }^{1}$, E Storm ${ }^{1}$, D J Strozzi ${ }^{1}$, L J Suter ${ }^{1}$, C A Thomas 1 , R P J Town ${ }^{1}$, E A Williams ${ }^{1}$, S V Weber ${ }^{1}$, P J Wegner ${ }^{1}$, D C Wilson ${ }^{3}$, K. Widmann ${ }^{1}$, C Yeamans ${ }^{1}$, A Zylstra ${ }^{5}$, J D Lindl ${ }^{1}$, L J Atherton ${ }^{1}$, W W Hsing ${ }^{1}$, B J MacGowan ${ }^{1}$, B M Van Wonterghem ${ }^{1}$, and E I Moses ${ }^{1}$

${ }^{1}$ Lawrence Livermore National Laboratory, Livermore, CA 94550, USA

${ }^{2}$ Laboratory for Laser Energetics, Rochester, NY, USA

${ }^{3}$ Los Alamos National Laboratory, Los Alamos, NM, USA

4Sandia National Laboratory, Albuquerque, NM, USA

5Plasma Science and Fusion Center, MIT, Cambridge, MA 02139, USA

${ }^{6}$ General Atomics, San Diego, CA, USA

${ }^{7}$ Atomic Weapons Establishment, Aldermaston RG7, UK

E-mail: hinkel1@llnl.gov

LLNL-CONF-641113

Progress toward ignition at the National Ignition Facility (NIF) has been focused on furthering the understanding of implosion performance. Implosion performance depends on the capsule fuel shape, on higher mode asymmetries that may cause hydrodynamic instabilities to quench ignition, on time-dependent asymmetries introduced by the hohlraum target, and on ablator performance. Significant findings in each of these four areas is reported. These investigations have led to improved in-flight capsule shape, a demonstration that a capsule robust to mix can generate high levels of neutrons $\left(7.7 \times 10^{14}\right)$, hohlraum modifications that should ultimately provide improved beam propagation and better laser coupling, and fielding of capsules with high-density carbon ablators. A capsule just fielded with an HDC ablator and filled with DT gas generated a preliminary record level of neutrons at $1.6 \times 10^{15}$, or $5 \mathrm{~kJ}$ of energy. Future plans include further improvements to fuel shape and hohlraum performance, fielding robust capsules at higher laser power and energy, and tuning the HDC capsule. A capsule with a nanocrystalline diamond (highdensity carbon, or HDC) ablator on a DT ice layer will be fielded at NIF later this year. 


\section{INTRODUCTION}

The prospect of laser fusion has tantalized scientists for decades. The process that fuels the sun and stars, where deuterium and tritium fuse to form a helium nucleus and release a $14.6 \mathrm{MeV}$ neutron, is now being sought in the laboratory, using the National Ignition Facility (NIF) [1]. The world's most powerful laser, NIF, is comprised of 192 laser beams, and provides up to 500 TW of laser power on target, and 1.8 MJ of laser energy after frequency tripling of $1.052 \mu \mathrm{m}$ light [2].

There are different approaches for achieving laser fusion. Currently, the inertial confinement fusion (ICF) program at NIF is primarily focused on indirect drive [35]. Here, the NIF laser beams enter a small $(\sim 1 \mathrm{~cm}$ in length, $0.575 \mathrm{~cm}$ in diameter $)$ high-Z cylindrical can (hohlraum) through an opening in the cylinder endcaps. These openings, or laser entrance holes (LEHs) are roughly half the diameter of the cylinder. With 96 laser beams coming in each LEH, the windows that hold the gas fill in the target blow down and the beams propagate to the hohlraum gold walls, where laser energy is converted to x-radiation (Fig. 1a). The interior of the hohlraum acts as a radiation oven that heats the capsule located in the center (Fig. 1b). The exterior region of the capsule is ablator material, typically plastic $(\sim 200 \mu \mathrm{m}$ thick). This ablator covers a frozen layer $(\sim 70 \mu \mathrm{m}$ thick) of deuterium (D) and tritium (T), which further encapsulates gaseous DT with a radius of $\sim 850 \mu \mathrm{m}$. As the capsule heats up, the ablator ablates, and the capsule compresses (through conservation of momentum, Fig. 1c). A hotspot forms in the gaseous center of the capsule, surrounded by a dense (frozen) DT shell. Fusion initiates in this central hotspot, and a burn front propagates outward [6] (Fig. 1d).

Not only does NIF have capability (in terms of power/energy), it also has the precision required to tune the capsule implosion [7]. By the end of 2012, NIF's careful pulse shaping enabled achievement of $85 \%$ of the areal density required for ignition [8] ( $\sim \mathrm{n} \tau$, Lawson criterion). Moreover, neutron yield was within $\sim$ a factor of 5 of the $\alpha$-heating regime. The requisite drive in excess of $300 \mathrm{eV}$ in the NIF hohlraums has also been achieved routinely [9].

Since that time, promising avenues to pursue have arisen $[10,11]$. The inertial confinement program (ICF) at NIF has recently been performing research on improved hohlraums, higher adiabat implosions, fielding capsules with a nanocrystalline diamond [or high-density carbon (HDC)] ablator, and diagnosing both low mode and high mode capsule asymmetries. This paper reports on progress in these arenas. The reader should be aware that this is a snapshot in time of the progress we have made, and that this report may very well be soon outdated.

Recent progress at NIF is focused on understanding the major factors affecting implosion performance [12]. These factors can be categorized into four different areas: shape, with the goal of keeping the implosion spherical; mix, with the goal of minimizing hydrodynamic instabilities [3, 13-16], where cold ablator mixes into the central hotspot and quenches ignition; hohlraum drive [9], with the goal of 
improving beam propagation and laser energy coupling [17, and references therein], and reducing time-dependent x-ray asymmetries; and alternate ablator implosions, with the goal of tuning implosions with HDC [18] ablators.

Within the category of shape, we have measured the in-flight capsule shape, and improved the shape [19]. These measurements suggest that time-dependent asymmetries may be introduced by the hohlraum, or, perhaps perturbations seeded by the capsule tent that holds the capsule in place within the hohlraum.

With respect to mix, we have found that capsule implosions fielded on a higher adiabat are more robust to mix, presumably because of a larger ablative stabilization effect, and because these capsules converge somewhat less than their companion low-adiabat capsule designs. These higher adiabat implosions achieved a primary (13-15 MeV) neutron yield of $7.7 \times 10^{14}$ on the first DT cryogenic implosion, a yield comparable to record yields for implosions on a lower adiabat [12].

At the writing of this report, NIF has fielded three shots with HDC ablators. These were not ignition capsules in that they did not have a DT ice layer inside of the ablator surrounding the DT gas. The first two shots were filled with DD gas, and the third shot with DT gas. The outer capsule radius was $\sim 1077 \mu \mathrm{m}$, as compared to $1135 \mu \mathrm{m}$ for a $\mathrm{CH}$ gas-filled capsule. The HDC ablator thickness is $\sim 77 \mu \mathrm{m}$ as compared to a $\mathrm{CH}$ ablator thickness of $\sim 195 \mu \mathrm{m}$. This leaves a larger DT gas volume in the HDC capsule, with a radius of $\sim 1 \mathrm{~mm}$ as compared to $850 \mu \mathrm{m}$ for a $\mathrm{CH}$ capsule. The third DT shot, fielded in late June, 2013, produced a preliminary value of $1.6 \times 10^{15}$ neutrons (+/- $6 \%$ uncertainty), or roughly $5 \mathrm{~kJ}$ of neutron yield. The HDC capsule for this shot was imploded on a low adiabat $(\sim 1.5)$ with a four-shock pulse.

In order for the capsule to perform well, the radiation environment that bathes it must not only meet the requirement of achieving a high enough temperature, but it must also be sufficiently symmetric. For this reason, we are looking to ameliorate hohlraum performance by improving laser beam propagation inside the hohlraum and by increasing laser energy coupling. We are doing this by testing the hohlraum shape (cylinder vs rugby [20-23]), and by developing schemes that are predicted to increase the electron temperature within the hohlraum. The former is predicted to have better beam propagation, and the latter should reduce laser reflectivity, thereby increasing coupling. Finally, we are also studying a potential source of timedependent asymmetries, cross-beam energy transfer [25, and references therein], which is routinely used at NIF to obtain near-round hotspot emission.

This paper is outlined as follows. In the next sections, we will discuss further the details of shape, then of mix, followed by hohlraum improvements, and then HDC implosions. The final section of this paper will summarize the progress at NIF and discuss future directions in each of these areas. 


\section{SHAPE}

Keeping the capsule spherically symmetric while it compresses 30-40x during an ignition implosion is a challenging endeavor. The NIF ignition capsule, initially at a diameter of $2 \mathrm{~mm}$, converges to the point where the diameter of hotspot emission is $\sim 50 \mu \mathrm{m}$. To this end, since experiments at NIF began in earnest in 2009, we have diagnosed the capsule hotspot emission, and have made the necessary adjustments to make the emission nearly round. The best performing shots, however, were not necessarily any rounder. The question thus arises, "what about the shape of the dense, cold shell that surrounds the hotspot?". To address this question, a new imaging technique has been fielded at NIF [19].

NIF now routinely detects the in-flight capsule shape, using this new imaging technique. Experiments are being conducted to backlight in 2D the capsule as a function of hohlraum length. The results of these experiments will be reported on in a separate publication.

In the future, the shape campaign will be looking at improving diagnostics to look later in time when the capsule is more compressed, and to also look earlier in time, when the capsule is less compressed. This campaign will also be researching timedependent symmetry, and the effect of imposing a deliberate asymmetry.

\section{MIX}

Just as low modes impact the fuel shape and thus capsule implosion quality, high mode surface perturbations act as seeds for hydrodynamic instabilities [13-16], which can mix cold ablator material (or, even worse, higher $\mathrm{Z}$ ablator material) into the capsule hotspot and quench ignition. One approach we have adopted is to field a capsule that is robust to such instabilities.

Fig. 2 depicts (in black) the radiation temperature (Tr) vs time for this capsule. It is a typical ignition drive [6], where each epoch in Tr launches a shock in the capsule. Shown in red in Fig. 2 is Tr vs time for a capsule driven with a stronger first shock. This stronger first shock introduces more entropy into the fuel and ablator. Since this first shock is traveling at a higher velocity, in order to maintain shock timing (so that the shocks merge just inside the ice-gas interface), the first epoch is shortened, and shocks two and three are merged. A capsule driven with this drive will not produce as much neutron yield (1D simulations produce 9 MJ of yield, vs 16-18 MJ for the drive shown in black). However, it is more robust to hydrodynamic instabilities. Ablative stabilization is greater, and the capsule also converges somewhat less because of its higher entropy.

Simulations of this high adiabat capsule (ratio of fuel pressure to Fermi degenerate pressure) predict that higher adiabat implosions have less ablation front and fuelinterface growth of hydrodynamic instabilities. In Fig. 3, we plot the maximum fuelablator interface growth factor (maximized over mode number) vs peak velocity for many simulations (both pre-shot and post-shot). The points depicted in purple are 
pre-shot simulations of the low-adiabat capsule, and those shown in green are postshot simulations. These points fall along an iso-adiabat $\alpha<\sim 1.55$. These points also exhibit a large max growth factor.

In some of these simulations, a deliberate shock mis-timing of the fourth shock was introduced. These simulations ended up on a higher adiabat $(\alpha \sim 2)$, and are depicted in blue. In these simulations, the max growth factor is reduced. Entropy has been added to the capsule, and it is not as compressible as when it was on a lower adiabat.

When a deliberate shock mis-timing is introduced earlier in the pulse (shock three overtakes shock two before they enter the DT gas), the adiabat increases to $\alpha \sim 3$, and the max growth factor is further reduced. These points are depicted in red. In general, a higher adiabat will lead to a lower density shell. The lower density will allow deeper penetration of the ablation surface, and thus more ablative stabilization of the RT instability.

When entropy is introduced through a strong first shock, and shocks are well-timed, we find that the max growth is even lower than when deliberate mis-timings are introduced. These points are results of simulations of the high-adiabat capsule described above. The max growth factor for hydrodynamic instabilities is 4-6 times less for the high-adiabat capsule when compared to the shock-timed low-adiabat capsule ( $\alpha \sim 1.55$ curve).

This high-adiabat capsule has recently been fielded at NIF (May 1, 2013). A comparison of neutron yield for this implosion is compared to the existing NIF database of ignition implosions in Fig. 4. Here, we plot experimental yield vs 1D simulated yield. The diagonal solid line is the curve along which the points would lie if the experimental yield matched the simulated yield. Dashed lines depict where experimental yield is at $50 \%, 20 \%, 10 \%, 5 \%$ and $2 \%$ of the simulated yield. The points shown in gray are gas-filled capsules. These capsules typically perform at about $50 \%$ of simulation.

The cluster of colored points depict ignition implosions, and are colored by the amount of inferred mix mass. Data points with high inferred mix mass do not perform as well as those with low amounts of inferred mix. At best, these lowadiabat implosions perform at $\sim 20 \%$ of simulation.

Our first high-adiabat ignition implosion achieved a primary (13-15 MeV) neutron yield of $7.7 \times 10^{14}$, a yield comparable to record yields for a low-adiabat implosion. The capsule mix mass (inferred from the ratio of x-ray emission from the capsule to neutron yield) is the lowest inferred to date. This high-adiabat ignition implosion not only achieved a relatively yield, but that yield was $\sim 80 \%$ of simulation, and was achieved at lower laser power and energy (350 TW/1.3 MJ) than in the bestperforming low-adiabat implosions (420 TW/1.5 MJ). The plan is to continue to 
increase laser power and energy until evidence of mix is observed. To this end, the next high-adiabat ignition implosion, planned for July, 2013, will occur at 430 TW and $1.5 \mathrm{MJ}$.

Since mix is clearly occurring in our ignition implosions, dedicated experiments to study instability growth as a function of mode number and adiabat are underway. In these experiments, ripples are purposefully imposed on a capsule, and single pass radiography is performed, resulting in time sequences of gated x-ray images of perturbation growth. The first test of the experimental set-up occurred early in June, 2013. A single mode (mode 60, which is near the peak of the growth factor curve) was imposed on the capsule, and snapshots were performed when the capsule had compressed to a radius $\mathrm{R} \sim 750 \mu \mathrm{m}$. Good data was acquired, and the next shot will be of a high-adiabat capsule at mode 60 . Results of these experiments will be reported later in the year at different venues.

\section{HOHLRAUM DRIVE}

The radiation drive that the capsule receives is that delivered by the hohlraum. NIF hohlraums produce the required radiation drive [9], but emerging evidence suggests that symmetry control of the radiation may not be adequate for ignition implosions.

In these hohlraums, outer beams (128 of 192) hit the hohlraum wall near the LEH, making angles of $44^{\circ}$ and $50^{\circ}$ with the hohlraum axis. Inner beams (64 of 192) travel through the hohlraum at angles of $23^{\circ}$ and $30^{\circ}$ with the hohlraum axis, striking the hohlraum wall over the capsule. Both the inner and outer beams fill the hohlraum azimuth as well.

To keep the imploding capsule round [rather than oblate ("pancaked") or prolate ("sausaged)], power must be distributed between the inner and outer beams properly. If there is too much energy on the inner beams, radiation over the waist of the capsule is hotter than that near the LEH, and the implosion will be prolate. If there is too little energy on the inner beams, radiation over the waist of the capsule is cooler than that near the LEH, and the implosion will be oblate.

NIF experiments in 2009 showed that the plasma conditions within the hohlraum are cooler than simulations indicated, primarily because the coronal plasma is more emissive than previously expected using less sophisticated atomic physics and electron transport codes, and thus is left with less stored internal energy [26]. This cooler electron temperature acts to increase the amount of inverse bremsstrahlung that inner beams undergo, which makes propagation over the entire path from the LEH to the hohlraum wall $(\sim 7 \mathrm{~mm})$ difficult.

Thus, to reduce oblate/prolate asymmetries in a time-integrated fashion, NIF uses laser-plasma interactions in the form of cross-beam energy transfer (CBET, [25, and references therein]). Here, energy is transferred using forward Brillouin scatter, where an outer beam forward scatters off an ion acoustic wave into an inner beam. 
Fig. 5a depicts a schematic of this process. Operationally, this is achieved by running the inner and outer beams at two different wavelengths: $\lambda$, and $\lambda-\Delta \lambda$, where $\Delta \lambda<\sim 9 \AA$.

Before undergoing power transfer, a laser beam is comprised of a super-Gaussian envelope filled with deliberately imposed laser speckles, as shown in Fig. 5b. An inner beam that receives transfer ends up with a spatially non-uniform distribution, as shown in Fig. 5c. This spatial non-uniformity arises because the inner and outer beams do not exactly overlap in the region where transfer occurs.

This spatial non-uniformity is also time dependent. Cross-beam energy transfer scales as $\sim I^{*} n_{e} / T_{e}$, where $I$ is the laser intensity, $n_{e}$ is the electron density, and $T_{e}$ is the electron temperature. Since the amount of transfer scales with all three of these quantities, and since they are all time dependent, the amount of transfer (i.e., how spatially non-uniform the laser spots are) will also be a function of time. Moreover, since the amount of transfer scales inversely with the electron temperature, which is lowest at the beginning of the pulse when the beams burn through the LEH window and hohlraum gas to the wall, there will also be spatially non-uniform laser spots early in the pulse.

The above explanation relies entirely on simulations. NIF experimental data corroborates the magnitude of the transfer, but we do not have experimental data about the spatio-temporal intensity dependence after transfer. To further investigate this phenomenon, we are developing an "LEH-only" target, or "quartraum".

In this target, depicted in Fig. 6a, the laser beams from one side (all 96) enter through the LEH just as they do in an ignition hohlraum. The beams therefore undergo cross-beam energy transfer experiencing a volumetric overlap and plasma conditions similar to those early in an ignition laser pulse. The outer beams strike the quartraum walls, producing radiation, and the inner beams travel through the quartraum and strike a detection plate. The plate emits radiation, which is experimentally diagnosed. If the contrast in the spatial non-uniformity across a beam spot is a factor of two or larger, as simulated (c.f. Fig. 6b) then the nonuniformity should be detectable. Quartraum experiments are planned for later this calendar year at NIF.

In addition to dedicated experiments that will elucidate CBET spatio-temporal dependences, we are investigating hohlraum modification options that would reduce the impact of such dependences. One such option is to change the hohlraum shape from a cylinder to "rugby". A hohlraum can be described by a Lame' curve, $(\mathrm{x} / \mathrm{A})^{\mathrm{n}}+(\mathrm{y} / \mathrm{R})^{\mathrm{n}}=1$, where $\mathrm{R}$ is the hohlraum waist radius, $\mathrm{L}$ is the hohlraum halflength, and $A=L\left[\left(1-R_{L E H} / R\right)^{n}\right]^{(-1 / n)}$. For a cylinder, $n=8.75$, and for the current NIF rugby shape, $n=1.8$. By changing the shape of the hohlraum from cylindrical (black) to rugby (blue), as depicted in Fig. 7a, the hohlraum wall area remains the same, but 
the diameter of the hohlraum over the capsule waist is larger for the rugby [20-24]. The former means that wall losses should remain the same in a rugby as they are in a cylinder. The latter is proposed to improve inner beam propagation. By providing more headroom over the capsule, ablated mass from both the capsule and the wall does not amass to the degree it does in a cylinder. With lower electron density, the beams should be able to propagate better. In fact, simulations prior to our first rugby hohlraum shot suggested that good symmetry was obtainable without any power transfer. [One can also make the radius of the rugby over the waist (above the capsule) comparable to that of a cylinder, and thereby reduce the total hohlraum wall area and thus wall losses. However, in the first rugby experiments at NIF, we want to improve inner beam propagation, and thus we keep the wall area the same as that of a typical NIC cylinder, and make the radius over the capsule larger.]

The first rugby shot at full power and energy demonstrated 93\% laser energy coupling, but the inner beams propagated poorly with minimal power transfer, resulting in an oblate hotspot. The current hypothesis is that the inner beams were absorbed in a larger-than simulated gold bubble that is produced where the outer beams hit the wall. To overcome this, our colleagues at CEA in France (P. Gauthier et al.) have recommended moving the outer beams inward $500 \mu \mathrm{m}$ (Fig. 7b). Their calculations suggest that by doing so, the inner beams will be able to better propagate to the wall over the capsule. They further suggest a slight modification to the shape of the rugby (Fig. 7c) that should further aid in inner beam propagation. These modifications to the geometry will be tested later this year and reported on in different venues.

We also want to increase laser energy coupling [17, and references therein] to the hohlraum. One possible way of doing this is to increase the electron temperature within the hohlraum. If the inverse bremsstrahlung, which scales with the ionization state, $\mathrm{Z}$, is increased, the electron temperature should increase as well.

To test this concept, we have been conducting a mini-campaign on hohlraums filled with neopentane gas. Since neopentane freezes out at cryogenic temperatures, these shots have been performed at room temperature. The effective ionization state for neopentane, or $\mathrm{C}_{5} \mathrm{H}_{12}$, as given by $\left\langle\mathrm{Z}^{2}>\right|<\mathrm{Z}>$, is $\mathrm{Z} \sim 4.6$, whereas for $\mathrm{He}, \mathrm{Z}=2$. Simulations show that, indeed, when the He hohlraum gas fill is replaced with neopentane at the same electron density, the electron temperature within the hohlraum increases, although somewhat marginally in the region along the inner beams where backscatter occurs, roughly halfway between the LEH and the hohlraum wall over the capsule. Fig. 8a depicts this electron temperature increase. This temperature increase should act to reduce backscatter that occurs along the inner beams, which is found to primarily be stimulated Raman scatter [17, and references therein]. Stimulated Raman scatter occurs when an incident laser beam scatters off self-generated Langmuir waves, and when the plasma electron temperature increases, the Langmuir waves are more strongly damped via Landau damping. 
Fig. 8b shows the laser energy coupling results from room temperature hohlraum shots. These shots were conducted at reduced levels of cross-beam energy transfer. The reflected light on the inner beams was reduced, but it is unclear whether this is a consequence of increased electron temperature or because less transfer was necessary to obtain a round implosion, or because of both reasons. Moreover, because less transfer was required, the intensity on the outer beams was higher (less power was transferred to the inner beams), and thus stimulated Brillouin scatter [17, and references therein], which scales in the linear regime with laser intensity, was increased. We know how to mitigate Brillouin backscatter, however. By introducing a thin layer $(<1 \mu \mathrm{m})$ of borated gold on the inside of the hohlraum wall, the Landau damping of ion acoustic waves associated with Brillouin backscatter will increase, and the SBS should be reduced. Even with larger levels of SBS on the outer beams, the energy coupling was increased from $87 \%$ to $92 \%$.

The challenge here is to try to increase the electron temperature in a cryogenic hohlraum. Here, only hydrogen and helium can be used, as all other gases will freeze out. We are investigating possible methods of introducing high-Z dopants in the helium hohlraum gas fill, and hope to make progress in this arena in the near future.

\section{ALTERNATE ABLATORS}

Another important aspect of the target is the capsule ablator. We have recently fielded three shots where the ablator was composed of nanocrystalline diamond (high-density carbon, or HDC), in lieu of plastic. HDC is more than three times denser than plastic, and its surface finish is smoother as well.

To test these benefits, capsules were fabricated as shown in Fig. 9a. These capsules are not ignition capsules in that they do not contain a frozen DT layer. Rather, they consist of an HDC ablator surrounding either DD or DT gas.

There is uncertainty in the equation-of-state (EOS) for HDC [27], and thus a conservative approach is to make certain that the first shock melts the HDC. In order to accomplish this, the first epoch of the laser pulse is at higher power than even the high-adiabat laser pulse for a plastic capsule (c.f. Fig 9b). To maintain shock timing, the length of the first epoch is further reduced, and thus the laser pulse for an HDC capsule is roughly half as long as for a low-adiabat plastic capsule.

The first two HDC shots were fielded with DD fill in the capsule. The first gave a yield of $1.06 \times 10^{13}$ neutrons, and the hotspot emission was quite prolate. In the second HDC shot, the hotspot shape was corrected by reducing the CBET (reducing $\Delta \lambda$ between the inner and outer beams), and the yield increased by $50 \%$ to $1.74 \mathrm{x}$ $10^{13}$ neutrons. In the third shot, the DD fill was replaced with DT, and achieved a preliminary record yield of $1.6 \times 10^{15}$ neutrons. Uncertainty in the yield is typically $+/-5-7 \%$. 
Fig. 9c summarizes the neutron yield results. The experimental yield was $\sim 70 \%$ of the simulated yield for the second HDC/DD-filled capsule, and was $\sim 80-100 \%$ of the simulated yield for the HDC/DT-filled capsule. The yield for this capsule should further improve after timing of the shocks and incorporation of a DT ice layer.

\section{SUMMARY}

ICF research at the National Ignition Facility has made good progress over the course of the last year. We have determined that the in-flight fuel shape was not round, and have made changes to the hohlraum length to correct the shape to nearround. Future plans in this area include imaging improvements of the in-flight fuel shape, and diagnosing the shape both earlier and later in time. The time-dependent symmetry of the capsule will be further examined, and the effect of imposing deliberate asymmetries will be pursued as well. The advent of high-energy, shortpulse laser technology for advanced radiography [28] will improve the accuracy of shape measurements as this capability becomes available

We found that capsules with plastic ablators driven on a higher adiabat perform close to 1D simulation predictions, providing a yield of $7.7 \times 10^{14}$ neutrons $(>80 \%$ of 1D simulated yield) when driven at a peak power of $350 \mathrm{TW}$ using $1.3 \mathrm{MJ}$ of laser energy. The robustness of these capsules to mix will be further tested this calendar year, by driving them at higher peak power and laser energy. Two-dimensional simulations suggest that yields up to $5 \times 10^{15}$ neutrons are attainable, provided that mix mass remains low. We plan to further study ablation front instability growth by performing dedicated mix experiments.

Just recently we also fielded rugby hohlraums, hoping to improve inner beam propagation and thus hohlraum performance. The first two rugby hohlraums shot at NIF had oblate hotspots. To correct this while remaining at minimal levels of cross-beam energy transfer, we plan to shoot a rugby hohlraum where the outer beams are moved inward $\sim 300 \mu \mathrm{m}$. We hope to also make a correction to the shape that should further improve beam propagation.

To improve laser energy coupling, we have looked at the prospect of increasing the electron temperature within the hohlraum by increasing the ionization state of the gas fill. Indeed, we have found that hohlraums with a higher-Z gas fill require lower levels of cross-beam energy transfer and have reduced levels of reflectivity. The challenge is to field a cryogenic hohlraum with higher-Z fill, which is an area of research we would like to pursue.

We will also field the quartraum platform to investigate the spatial non-uniformity of cross-beam energy transfer. If this is successful, the quartraum may be used to address early time physics such as specular reflection onto the capsule from the hohlraum walls.

We have also fielded targets at NIF with an HDC ablator. A DT-filled gas capsule with an HDC ablator achieved a preliminary record yield of $1.6 \times 10^{15}$ neutrons. A 
mini-campaign to tune an HDC implosion is underway, and we hope to field a capsule with an HDC ablator on a DT ice layer later this calendar year.

Lastly, during the final edit of this paper, a HiFoot DT implosion was fielded on NIF on September 27, 2013, using 380 TW of laser power and 1.8 MJ of laser energy. This implosion achieved a primary (13-15 MeV) neutron yield of $4.3 \times 10^{15}$, which is well on the way to the alpha heating regime. The details of this shot will be reported on in several upcoming venues.

\section{Acknowledgments}

The authors would like to acknowledge the valuable input of the NIF laser and target diagnostic scientific and operations teams as well as that of the LLNL and GA target fabrication and assembly teams. This work was performed under the auspices of the US Department of Energy by Lawrence Livermore National Laboratory under Contract DE-AC52-07NA27344. 


\section{REFERENCES}

[1] E. I. Moses and C. R. Wuest, 2005 Fus. Sci. Tech. 47314.

[2] C. A. Haynam, P. J. Wegner, J. M. Auerbach et al., 2007 Appl. Opt. 463276.

[3] J. H. Nuckolls, L. Wood, A. Theissen, and G. B. Zimmerman, 1972 Nature 239139.

[4] J. D. Lindl, Inertial Confinement Fusion (Springer-Verlag, New York, 1998), pp. 1-15.

[5] Stefano Atzeni and Jurgen Meyer-ter-Vehn, The Physics of Inertial Fusion, International Series of Monographs on Physics (Oxford University Press, New York, 2004).

[6] S. W. Haan, J. D. Lindl, D. A. Callahan et al., 2011 Phys. Plasmas 18051001.

[7] H. F. Robey, P. M. Celliers, J. L. Kline et al., 2012 Phys. Rev. Lett. 10821504.

[8] O. L. Landen, R. Benedetti, D. Bleuel et al., 2012 Plasma Phys. Control. Fusion 54 124026.

[9] J. L. Kline, S. H. Glenzer, D. A. Callahan et al., 2013 Phys. Plasmas 20056314.

[10] Report from the Workshop on the Science of Fusion Ignition on NIF, https://lasers.llnl.gov/workshops/science_of_ignition

[11] ICF Program Execs Report to Congress, October, 2012, http://www.trivalleycares.org/new/NIF-

ICFProgramExecsReporttoCongressOctober2012.pdf

[12] M. J. Edwards et al., 2013, Phys. Plasmas 20, 070501.

[13] Lord Rayleigh, 1883 Proc. London Math. Soc. 14170.

[14] I. G. Taylor, 1950 Proc. R. Soc. London Ser. A 201192.

[15] R. D. Rychtmyer, 1960 Commun. Pure Appl. Math. 13297.

[16] V. Andronov, S. M. Bakhrakh, E. E. Meshkov, V. N. Mokhov, V. V. Nikiforov, A. V. Pevnitskii, and A. I. Tolshmyakov, 1976 Sov. Phys. JETP 44424. 
[17] D. E. Hinkel, M. D. Rosen, E. A. Williams et al., 2011 Phys. Plasmas 18056312.

[18] Peter Amendt, C. Cerjan, A. Hamza, D. E. Hinkel, J. L. Milovich, and H. F. Robey, 2007 Phys. Plasmas 14056312.

[19] R. Rygg et al., to be submitted to Phys. Rev. Lett., Summer, 2013.

[20] Peter Amendt, C. Cerjan, D. E. Hinkel, J. L. Milovich, H.-S. Park, and H. F. Robey, 2008 Phys. Plasmas 15012702.

[21] F. Philippe et al., 2010 Phys. Rev. Lett. 104035004.

[22] H. Robey et al., 2010 Phys. Plasmas 17056313.

[23] M. Vanderboomgaerde et al., 2007 Phys. Rev. Lett. 99065004.

[24] S. Laffitte et al., 2004 Phys. Plasmas 17102704.

[25] P. Michel, S. H. Glenzer, L. Divol, D. K. Bradley, D. Callahan, S. Dixit, S. Glenn, D. Hinkel, R. K. Kirkwood, J. L. Kline, W. L. Kruer, G. A. Kyrala, S. Le Pape, N. B. Meezan, R. Town, K. Widmann, E. A. Williams, B. J. MacGowan, J. Lindl, and L. J. Suter, 2010 Phys. Plasmas 17056305.

[26] Rosen, M.D., Scott, H.A., Hinkel, D.E., Williams, E.A., Callahan, D.A., Town, R.P.J., Divol, L., Michel, P.A., Kruer, W.L., Suter, L.J., London, R.A., Harte, J.A., and Zimmerman, G.B., 2011 HEDP 7180.

[27] D. K. Bradley, J. H. Eggert, D. G. Hicks, P. M. Celliers, S. J. Moon, R. C. Cauble, and G. W. Collins, Phys. Rev. Lett. 96195506.

[28] C. P. J. Barty, M. Key, J. Britten et al., 2004 Nuclear Fusion 44 S266. 


\section{FIGURE CAPTIONS}

Fig. 1 The processes entailed in indirectly driven laser fusion. (a) Coupling: laser energy enters the hohlraum target, which encloses a pellet of DT encased in ablator material, strikes the hohlraum walls, and is converted to x-radiation. (b) Drive: the $\mathrm{x}$-radiation fills the hohlraum, creating a radiation oven that bathes the capsule, and the ablator heats up. (c) Symmetry: the ablated shell expands outward, and the remainder of the capsule is compressed inward (conservation of momentum). If the capsule does not remain nearly spherical while being compressed, it will not achieve conditions required for fusion initiation. (d) Fusion initiates in a central hot spot where the ion temperature is high, and a burn front propagates outward.

Fig. 2 Radiation temperature vs time for a low-adiabat capsule (in black) vs a highadiabat capsule (in red).

Fig. 3 A plot of the maximum growth factor at the fuel-ablator interface vs peak velocity for ignition implosions driven on a low adiabat $(\alpha \sim 1.55$, points in purple and green); for implosions with mis-timed shocks, that then end up on a higher adiabat ( $\alpha \sim 2, \sim 3$, in blue and red); and for implosions on a high adiabat with good shock timing $(\alpha \sim 2.5$, black). The maximum growth factor for high adiabat implosions (black) is 4-6 times lower than that of a low adiabat implosion (purple/green).

Fig. 4 A comparison of neutron yield across a suite of shots. Here we plot experimental yield vs 1D simulated yield. Ignition implosions are colored according to their amount of inferred mix mass. The high-adiabat ignition implosion has the highest yield of an implosion with a DT ice layer, and the experimental yield was $80 \%$ of the 1D simulated yield, with low mix mass (inferred from the ratio of capsule $\mathrm{x}$-ray emission to neutron yield). The solid line is where experimental yield is $100 \%$ of simulated yield (righthand vertical axis). The dashed lines below the solid line refer to percentage of experimental yield compared to simulated yield. Shown in gray is the yield from capsules filled with D-He3 gas inside of a $\mathrm{CH}$ ablator (no DT ice layer).

Fig. 5 (a) To control P2 symmetry in a time-integrated fashion, NIF uses crossbeam energy transfer, where an outer beam forward scatters off and ion acoustic wave into an inner beam. (b) A NIF laser beam before it undergoes cross-beam energy transfer is composed of deliberately imposed laser speckles, with a spatially uniform envelope. (c) A NIF laser beam after it has traversed the LEH and undergone power transfer has a spatially non-uniform distribution, as power is not transferred uniformly across its cross section.

Fig. 6 (a) To investigate the spatial non-uniformity of laser spots after power transfer, the quartraum platform is being developed. (b) Simulations predict that 
the spatial non-uniformity across a laser spot has a contrast $\sim 2$ or greater. We propose to diagnose the non-uniformity by letting the inner beams strike a detection plate that emits radiation.

Fig. 7 We are investigating changes in the hohlraum geometry to improve inner beam propagation and laser energy coupling. (a) A rugby hohlraum with the same wall area as a cylinder is predicted to improve inner beam propagation because of the increased headroom at the capsule waist. (b) Moving the outer beams $300 \mu \mathrm{m}$ inward should improve rugby performance. (c) A slight change to the shape of the rugby is also predicted to improve performance.

Fig. 8 (a) Simulations suggest that a high-Z gas fill increases the electron temperature. (b) Laser energy coupling is reduced to $8 \%$ from $13 \%$ by increasing the $\mathrm{Z}$ of the hohlraum gas fill, while keeping the electron density the same.

Fig. 9 (a) Pie diagram of a capsule with an HDC ablator that is filled with either DD or DT gas. (b) The laser pulse for the HDC implosion is half the length of that for a low-adiabat $\mathrm{CH}$ capsule. (c) A DT-filled, HDC capsule just recently achieved a preliminary record yield of $1.6 \times 10^{15}$ neutrons, at $\sim 80 \%$ of what $1 \mathrm{D}$ simulations predict. Ignition implosions are colored according to their amount of inferred mix mass. Shown in gray is the yield from capsules filled with $\mathrm{D}-\mathrm{He} 3$ gas inside of a $\mathrm{CH}$ ablator (no DT ice layer). 


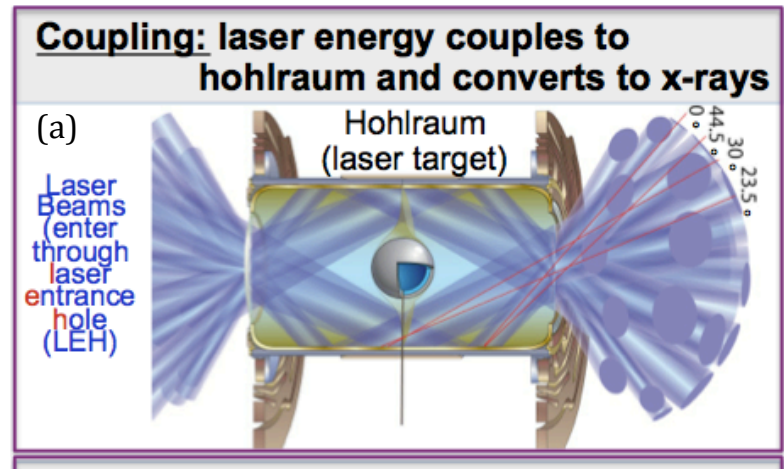

Symmetry: radiation compresses capsule and it implodes

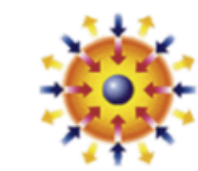

Rocket effect

- conservation of momentum: ablated shell expands outward, rest of shell (frozen DT) is forced inward
Drive: $x$-rays bathe capsule, heating it up -- it expands

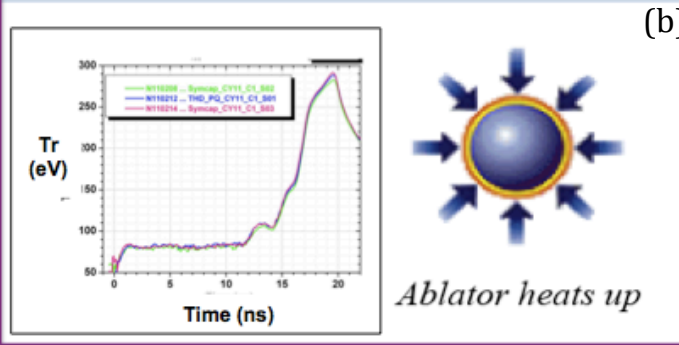

Fusion initiates in a central hot spot and a burn front propagates outward
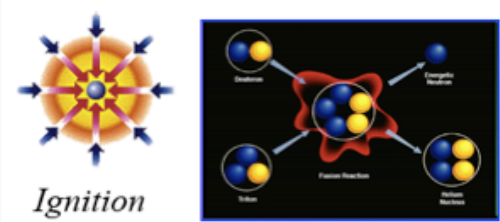

(c)

(d)

Fig. 1 


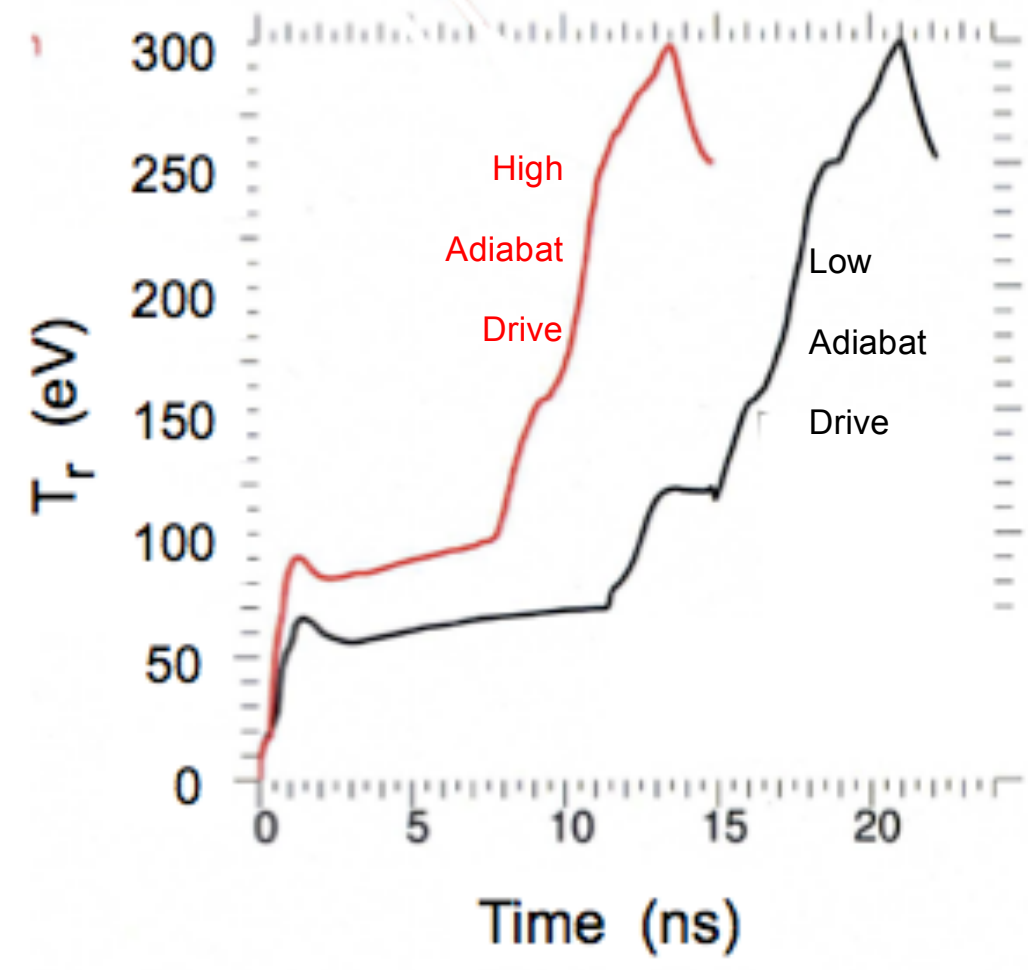

Fig. 2 


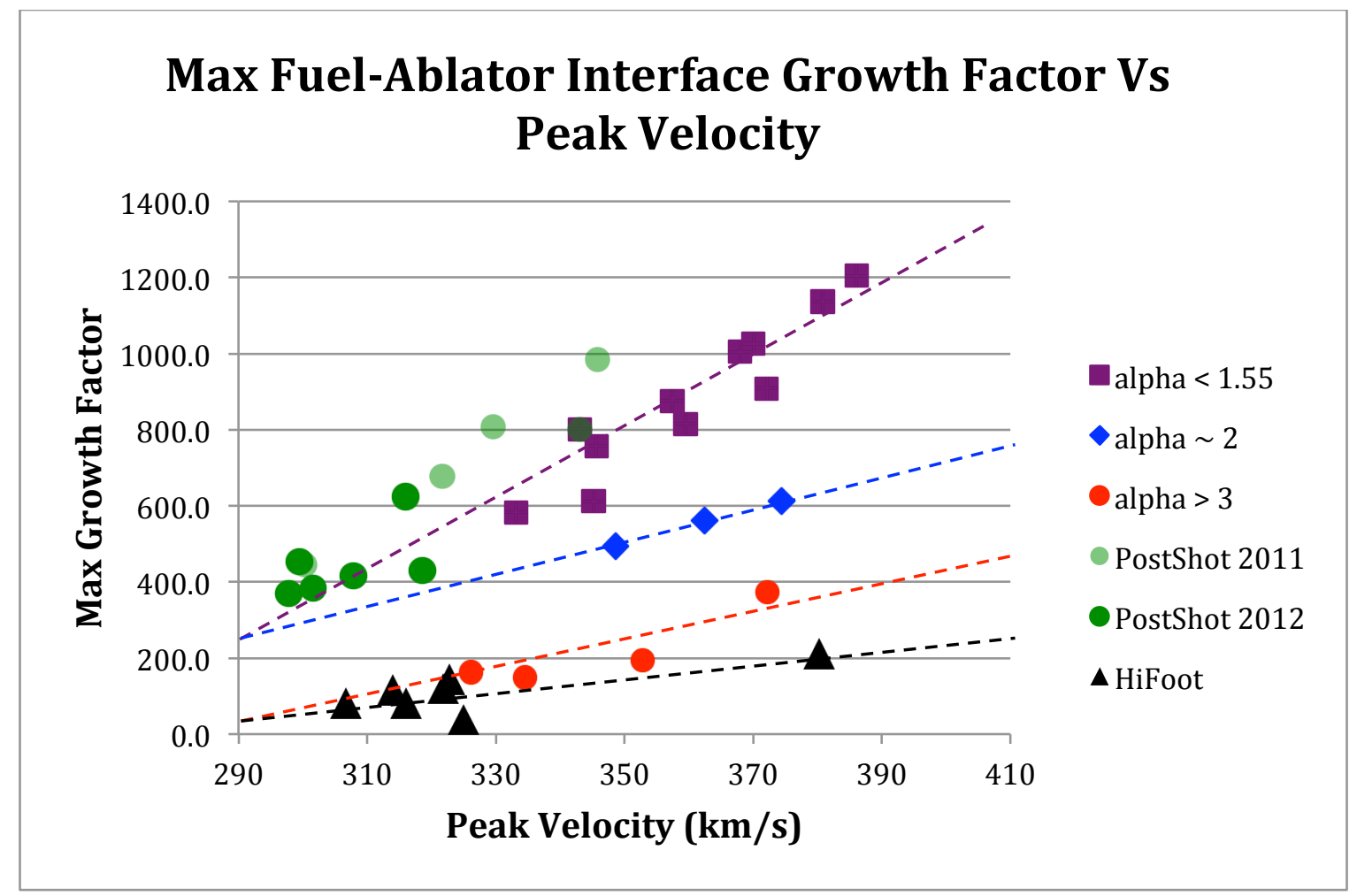

Fig. 3 
Neutron Yield

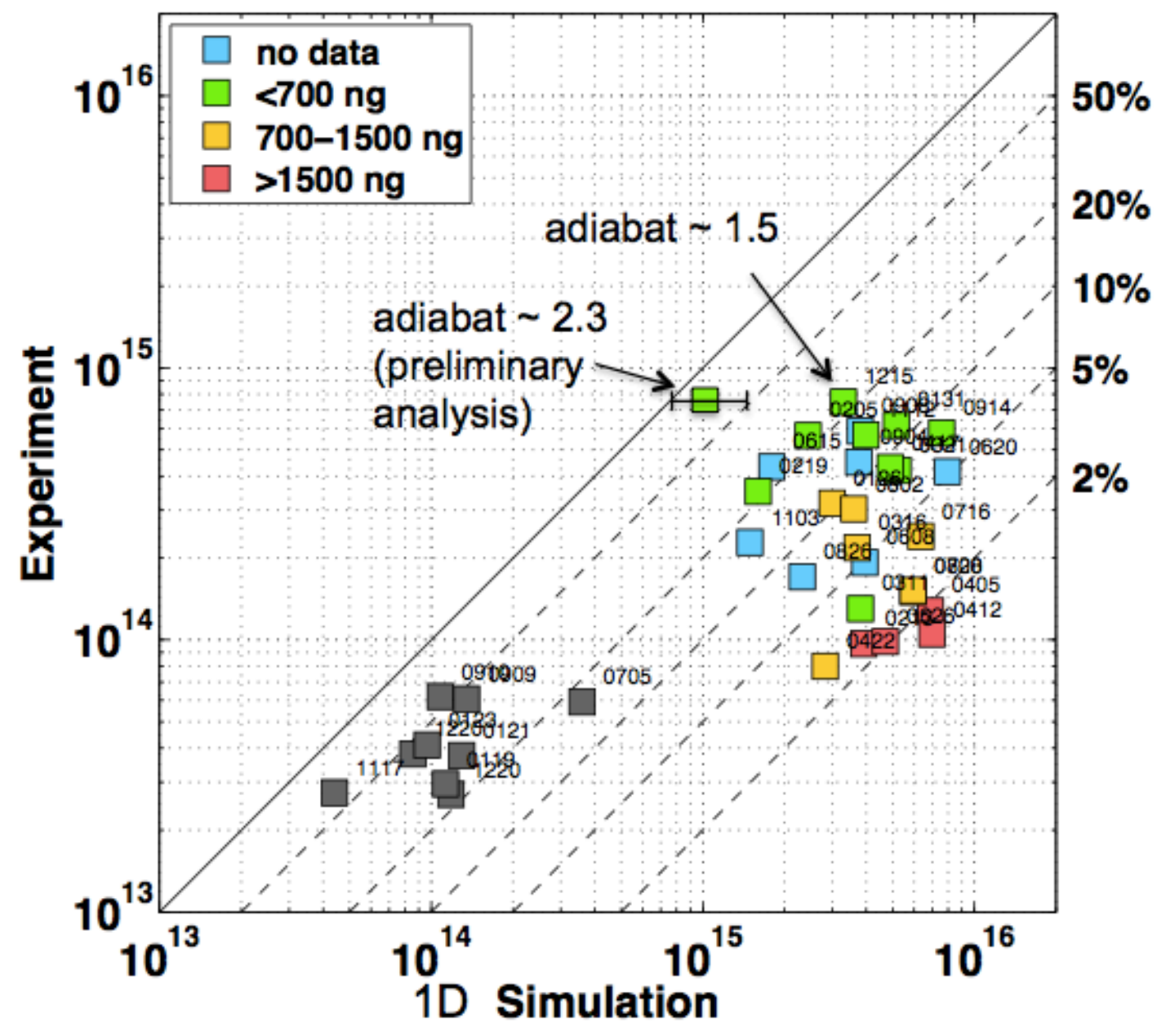

Fig. 4 

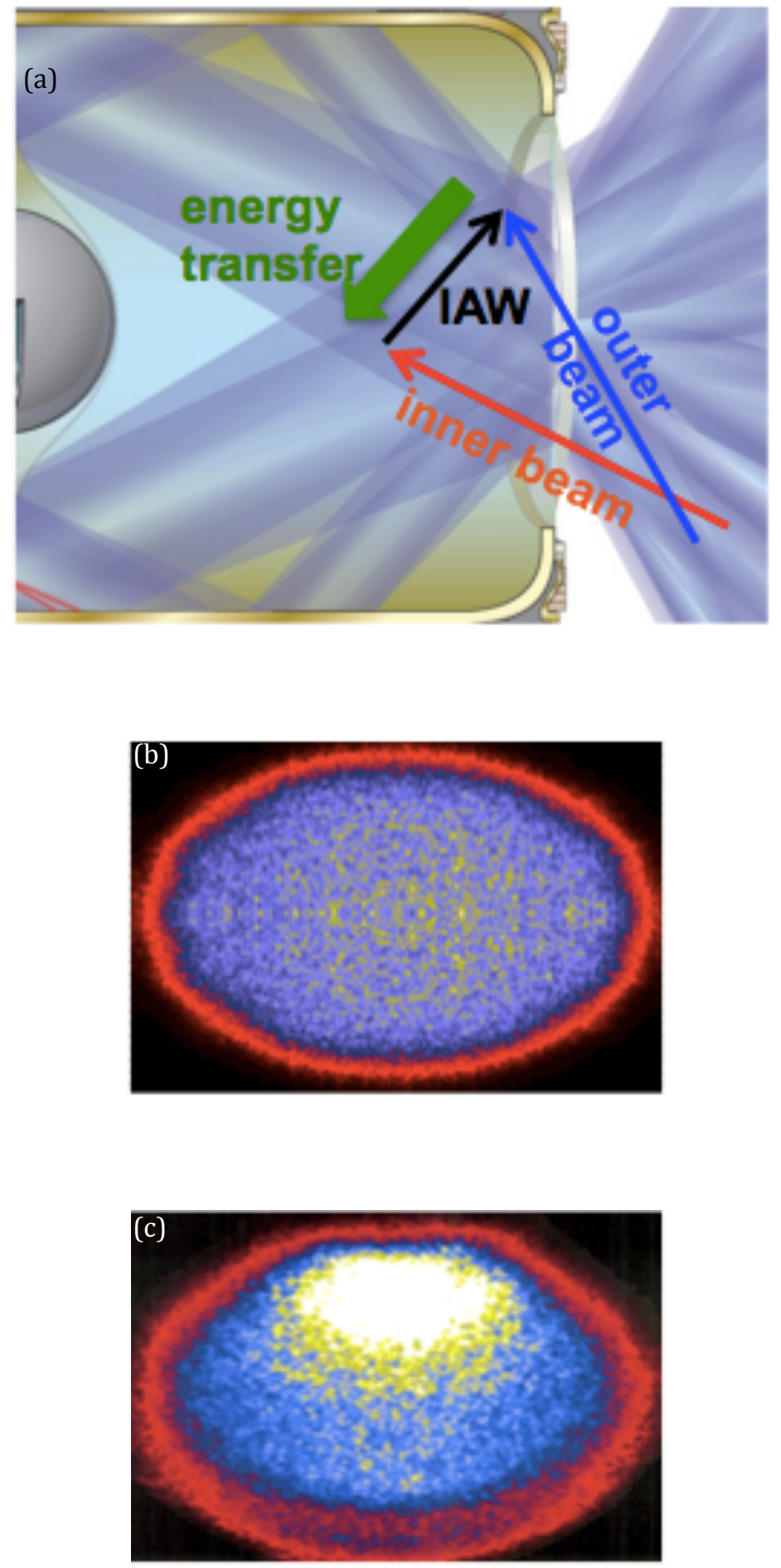

Fig. 5 

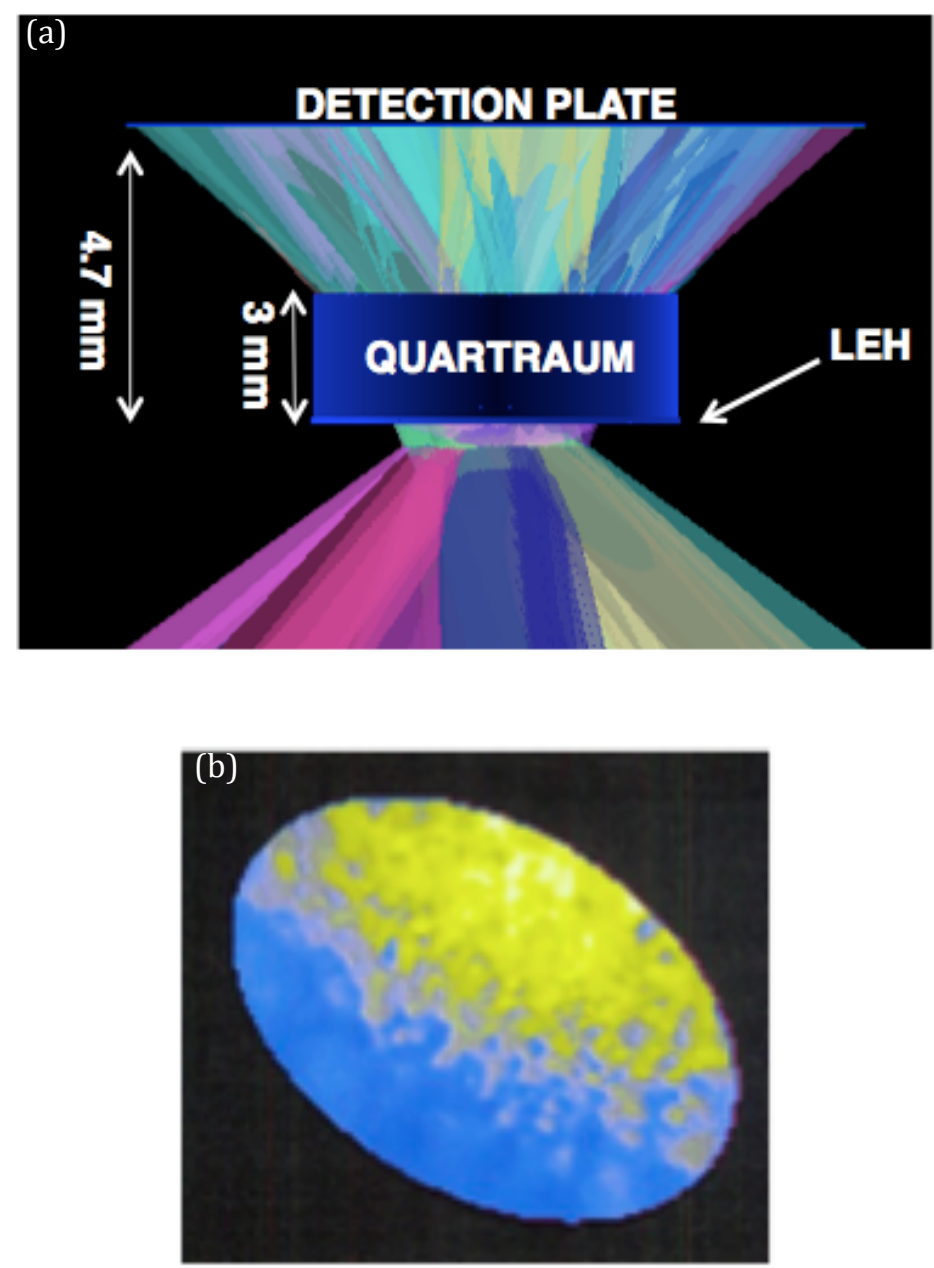

Fig. 6 
(a)

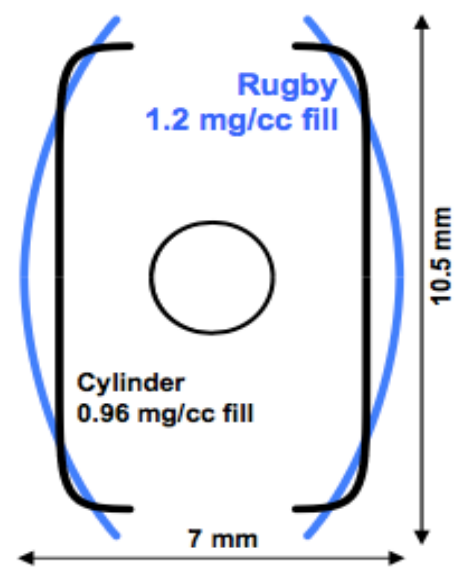

(b)

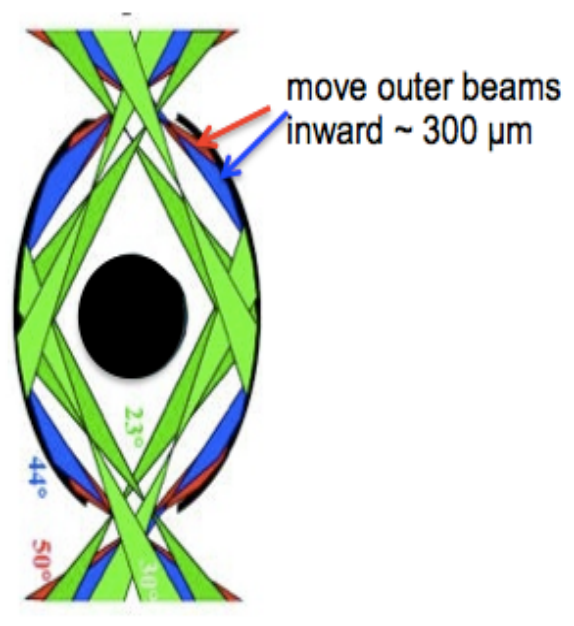

(c)

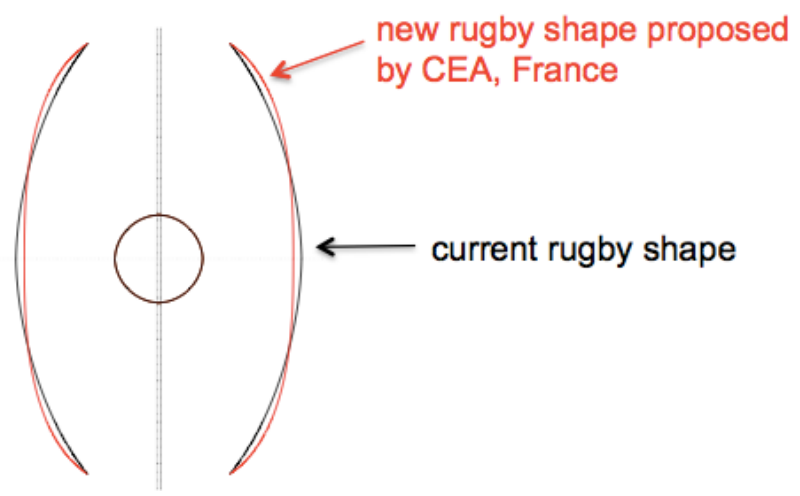

Fig. 7 
(a)

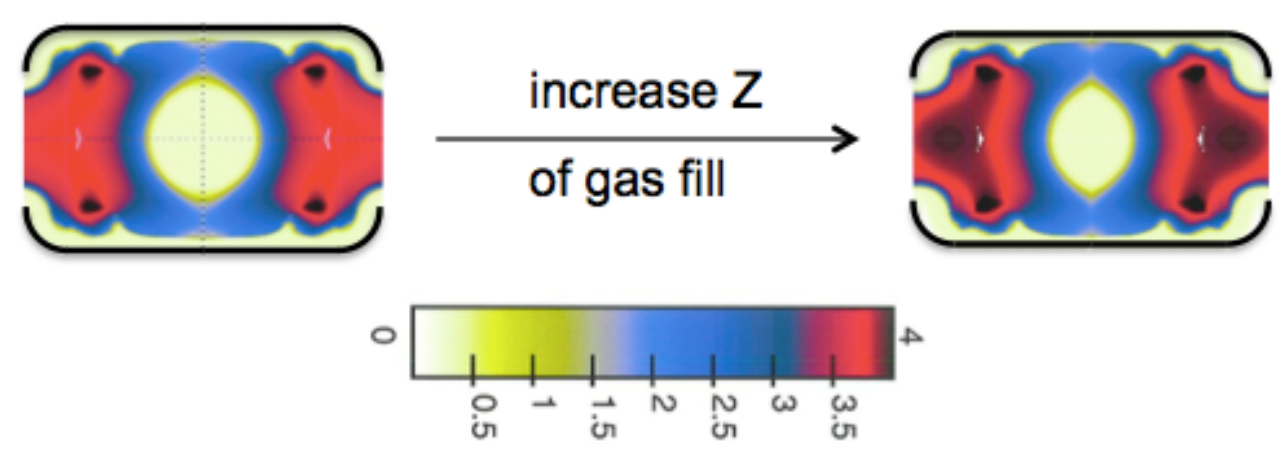

(b)

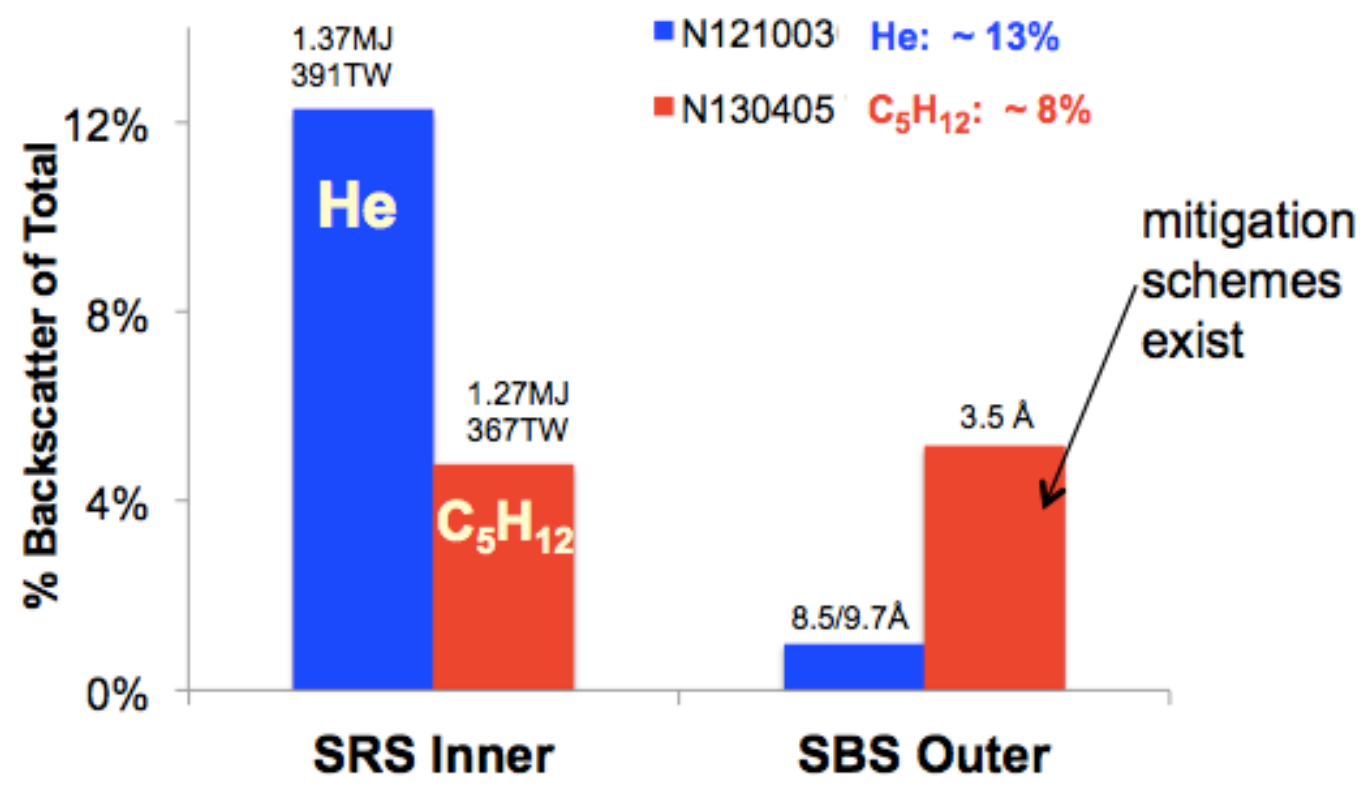

Fig. 8 
(a)

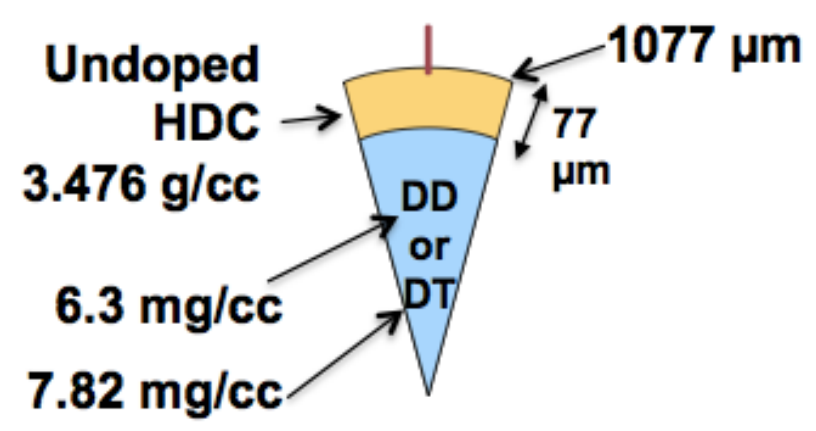

(b)

(c)
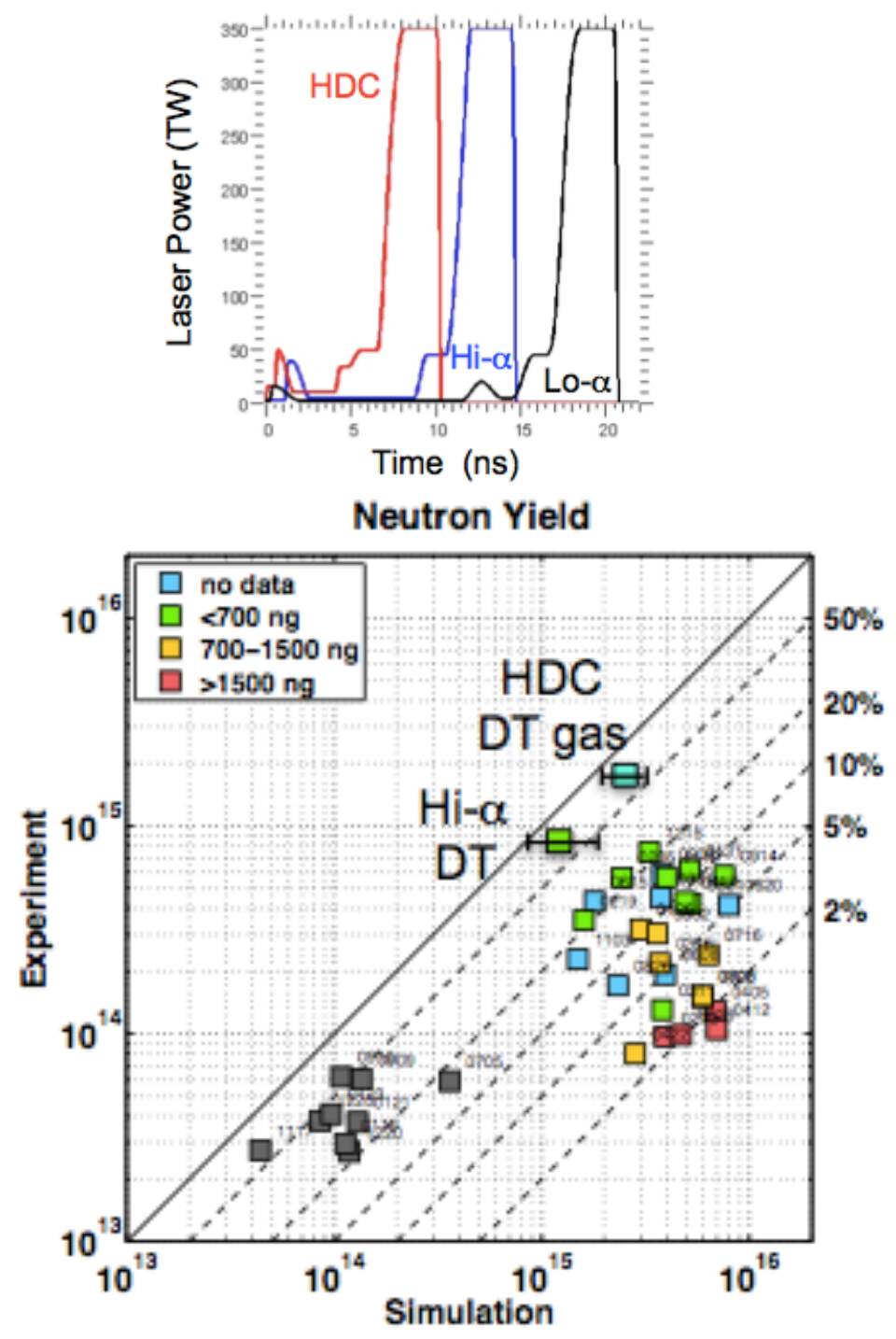

Fig. 9 